\title{
LA INTEGRACIÓN DE LAS TECNOLOGÍAS DE LA INFORMACIÓN Y LA COMUNICACIÓN EN LA FORMACIÓN DEL PROFESORADO: DESAFÍOS PARA ENSEÑANZA Y APRENDIZAJE CONSTRUCTIVISTA
}

\author{
Isabel Álvarez \\ Universidad Autónoma de Barcelona, España \\ Brent Kilbourn \\ Universidad de Toronto, Canadá
}

\section{INTRODUCCIÓN}

De qué manera se puede enseñar a los alumnos de magisterio a utilizar las TIC con la intención de poder integrarlas en las aulas, tiende a ser una problemática que no carece de actualidad. En este artículo nos planteamos si la respuesta es afirmativa, entonces deberíamos aproximarnos desde un enfoque integrador, a la vez que significativo, del currículo tan pronto como se pueda, es decir, desde su Formación Inicial. Sin embargo, muchas veces ocurre que estos alumnos no disponen de experiencia docente y tienden a ser tecnófobos, agravando la situación el encontramos delante de un enfoque transmisivo de los modelos de enseñanza y aprendizaje, entonces nos planteamos de qué manera se les puede ayudar a pensar de forma creativa sobre las potencialidades de integrar las TIC en el aula?

En este artículo describimos nuestras experiencias, tanto positivas como negativas a lo largo de estos años en la enseñanza universitaria. (Se trata de una investigación cualitativa que implica análisis de nuestros alumnos así como de la literatura al respecto.) El curso que queremos presentar se sitúa dentro del programa de formación de profesorado en una universidad Española. A lo largo de estos años nuestro interés ha ido en aumento al implicar a los alumnos en la construcción de páginas web mediante el Aprendizaje Basado en Problemas (CTGV, 1993) y la práctica reflexiva (Schön, 1983, 1987). Hemos trabajado con los alumnos la construcción de lo que hemos llamado webs integradoras. Este tipo de páginas (Álvarez y Kilbourn, 2003, 2004), que discutiremos a continuación, se caracterizan por (a) su función educativa coherente, (b) su construcción, permite ofrecer un ejemplo de integración dentro del currículo escolar, y (c) por hacer partícipes tanto a los profesores como a los alumnos durante todo el proceso. Además de aprender los habilidades propiamente tecnológicas, el proceso de construcción de páginas web es una manera de aprender contenido educativo. Nuestro objetivo es el de poder aumentar las posibilidades para que los alumnos utilicen las TIC cuando se enfrenten a sus cursos (futuros) en los centros educativos. Por lo que hace referencia al curso en sí, decir que nuestro objetivo es el de hacerlos participar en una experiencia para estimular de manera, lo mas realista posible, lo que representa una clase ordinaria, al mismo tiempo que perseguimos el ayudarlos a reflexionar sobre su propio proceso de aprendizaje de manera colaborativa.

La tarea de enseñar a los alumnos que no disponen de una experiencia docente previa y que al mismo tiempo se sienten incómodos con el tipo transmisivo de enseñanza/aprendizaje hace que el resultado sea desafiante. Durante los años que hemos podido trabajar con los alumnos en la formación del 
profesorado, hemos documentado y reflexionado sobre nuestra propia pedagogía, articulando aquellos elementos que han funcionado con los que no. Desde nuestra experiencia, las dificultades que hacen difícil el poder integrar las TIC de manera efectiva en el currículo vienen como consecuencia de; la persistencia de un modo transmisivo de la enseñanza/aprendizaje y la ausencia de experiencias docentes vividas por parte de los alumnos. Es por ello que queremos trabajar estos dos elementos en este articulo. Proponemos un ejemplo de uso de las TIC que es integrador, utilizando los principios constructivistas, para poder discutir los elementos contextuales que hace que nuestras propuestas sean desafiantes. Empecemos, pues por plantear de forma genérica el contexto tal y como lo vemos.

\section{EL CONTEXTO DE CONSTRUCCIÓN Y TRANSMISIÓN}

La polaridad entre transmisión y constructivismo reaparecen continuamente en la educación superior, incluyendo como preparamos a nuestros futuros profesores. De manera global, se puede decir que se utilizan diferentes términos para denotar la formación del profesorado siendo sus significados representativos de lo que queremos llegar a decir (no siempre coincidiendo con la misma visión del aprendizaje). La visión transmisiva indica que hay unas habilidades que el profesor necesita adquirir al mismo tiempo que éstas son las que necesitan ser transmitidas en su formación; es entonces cuando el futuro profesor está preparado para enfrentarse a la complejidad del aula. Es ésta tradición la que relacionamos con un enfoque mecanicista, debido a la repetición de unas habilidades hasta que ellas se han adquirido de manera inequívoca. Al contrario, una visión constructivista no pretende que la adquisición de habilidades o comprensión de un realidad se base en su mera abstracción, (eso caería en oídos sordos del profesor), el cual a su vez, muchas veces no dispone de experiencias docentes previas con las cuales conectar la formación para poder convertirla en algo significativo para su futuro profesional docente. Esta visión es la que comparamos con un enfoque organicista ya que entre otras cosas, ve que la formación del profesorado como un proceso que no termina con su formación inicial universitaria sino que se produce a lo largo de la vida, es un aprendizaje constante y que es, a su vez necesario disponer de herramientas con las cuales poder reflexionar y elaborar conocimiento.

La relación entre enseñanza y el aprendizaje refleja las tensiones que hay en la formación inicial del profesorado. El aprendizaje de las TIC se puede contemplar como el adquirir unas habilidades para después ser utilizadas en la práctica, mientras, que desde otra visión este aprendizaje podría ser visto como aquellas situaciones reales donde hay una simbiosis entre las finalidades educativas y las tecnológicas, es decir, unas necesitan a las otras. El enfoque que enfatiza una aproximación más realista de la utilización de estas habilidades en un contexto real, hace que tenga más posibilidades que se produzca, después, la integración por parte del profesor en su propio curso, sin embargo el centrarse exclusivamente en la integración necesita ir más allá. Un enfoque más orgánico, donde se reconoce el hecho de adquirir ciertas habilidades pero se contextualiza con la complejidad real de los centros y ayuda a la formación inicial a debatir cuales son los tipos de elementos que se pueden beneficiar de un uso comprensivo de las TIC; este enfoque es con que queremos plantear este artículo.

De manera global se sabe que la integración de las TIC en los centros no ha seguido el proceso que uno hubiera querido anticipar. Por ejemplo, Franklin, et al (2001: 26-27) describen cinco barreras que impiden su completa integración: 
- Visión. A la mayoría del profesorado le cuesta ver como se pueden llegar a integrar las TIC en el currículo escolar (Ertmer,1999; OTA, 1995);

- Acceso. A pesar de la incorporación de la tecnología en los centros, todavía es muy precaria la ayuda para poder disponer de ordenadores e infraestructuras en condiciones óptimas de trabajo (Ertmer, 1999; Fabry \& Higgs, 1997);

- Tiempo. El profesorado necesita tiempo para experimentar con los ordenadores, compartir experiencias con otros colegas, planificar nuevamente sus programaciones para incorporar las tecnologías, y sobretodo necesitan formación permanente en el uso de las TIC (Ertmer, 1999; Fabry \& Higgs, 1997);

- Evaluación. El profesorado necesita aprender nuevas estrategias para evaluar los resultados que provienen de la incorporación de las TIC en las aulas (Ertmer, 1999; OTA, 1995);

- Formación del Profesorado. Más veces de lo que quisiéramos, los cursos que se ofrecen para su formación se centran en el uso de una herramienta pero pocas veces en como ésta se puede integrar en el currículo.(Brunner, 1992; Ertmer, 1999; Fabry \& Higgs, 1997, OTA, 1995; Haugland, 1999)

Es importante detenerse en éste último punto descrito por Franklin sobre la Formación del Profesorado (tanto Inicial como Permanente). La Formación del Profesorado es más compleja que los aspectos técnicos así como infraestructuras o recursos disponibles (aunque ellos también son relevantes). El hecho de que, más a menudo de lo que quisiéramos, se considera a las tecnologías desde un punto de vista mecanicista es por (1) la ejercitación de una habilidad específica (email, html, powerpoint), y (2) cuando se enseña, se la presenta de manera aislada con el contexto al que va dirigido, en este caso, el curriculum. Estos enfoques más mecanicistas no aprovechan el potencial que puede llegar a tener si nos dirigimos a la complejidad donde se va a desarrollar. Según Salomon (2000), hay la tendencia en el sistema educativo de,

[...] preservarse en sí mismo así como sus prácticas cuando se presenta un nuevo recurso y en el mantener el todo sin cambios significativos. De este manera la tecnología se presenta como "domesticada" lo que realmente significa, se le permite operar dentro de la filosofía educativa de la transmisión cultural. ${ }^{1}$

Una de las muchas razones por las cuales el integrar las TIC en el curriculum de manera significativa no deja de ser un desafío muy importante, es el hecho de que no todos los profesores en un su formación han podido disfrutar de ejemplos. Así Zhao, et al (2002:511) destacan

[...] La mayoría de estos esfuerzos tienden a tomar una visión reducida de cómo el profesorado necesita utilizar la tecnología- habilidades y buena actitud.

Una razón para poder explicar el porqué es difícil ésta integración es el hecho de que no han tenido la oportunidad de experimentar a lo largo de su formación. No pudieron ver diferentes formas de integración pudiendo ésta ser más significativa y enriquecedora ${ }^{2}$. Este hecho hace que la raíz del problema se

\footnotetext{
${ }^{1}$ Hay diversos ejemplos de la observación que hace Salmon y uno de ellos, es la herramienta Knowledge Forum (Bereiter and Scardamalia, 2000). Este tipo de herramientas tienden a ser excepciones de este tipo de reglas.

${ }^{2}$ Varios autores han trabajado con los problemas de la integración de las TIC en la formación del profesorado: Becker, 1994; Beichner, 1993; Brand, 1997;Brush, 1998; Brush, T., et al., 2001; Carr, 1992; Duhaney, 2001; Fabry and Higgs, 1997; Falba, et al. 1999; Franklin, T. et al., 2001; Martínez and Woods, 1995; Munday, et al., 1991; Northoever, 1999; OTA, 1995; Pierson and McNeil 2000; Rodriguez, 1996; Schrum, 1999; Siegel, 1995; Smith, Houston, \& Robin, 1994; Strudler and Wetzel, 1999; Sugar, 2002; Thompson, Schmidt, \& Hadjiyianni, 1995; Topp et al, 1995; Wetzel, 1993; Willis, 1997.
} 
encuentre en su Formación. Brush, et al (2001:17), presentan su propia institución, basándose en la idea de que los requisitos en muchos de estos programas de formación del profesorado son problemáticos por,

La ausencia de integración entre la enseñanza de metodología en que se basan en experiencias con la enseñanza de la integración de las TIC con experiencias... La ausencia en la integración de la tecnología con experiencias reales... La ausencia de la integración entre los profesores del programa de formación de manera global.

Este tipo de problemática descrita por Brush (2001) y Zhao (2002) converge con la idea que presentamos en este artículo de que la formación del profesorado necesita ejemplos de prácticas reales e integradoras que ayuden a los alumnos y que sirvan para pensar más allá de unas habilidades especificas con una herramienta en particular. Las características de una visión mecanicista no dejan de aflorar en la superficie y en la manera de cómo estos futuros profesores pueden llegar a pensar de otra forma. Nuestra aportación es que manteniendo esta visión mecanicista hace que nos limitemos mucho y que necesitemos otra manera para ampliar perspectivas y planteamientos.

Un ejemplo ilustrativo de las limitaciones de una visión mecanicista es la noción de Internet, la cual es esencialmente orgánica. Esto quiere decir, que los conceptos orgánicos de integración, conexión, coherencia interna, son element os centrales de cómo funciona Internet. La interconexión compleja entre las distintas redes operativas, el hecho de que permite la conexión de la información (de varios tipos) en cualquier momento independientemente de donde se encuentre la información de manera física. Al mismo tiempo las diferentes redes están distribuidas globalmente pero no por ello significa que sean independientes. Cada red se puede contemplar como una porción diminuta en términos orgánicos. Aunque cada porción no se encuentra de manera aislada sino que forma parte de un todo muy complejo. De esta manera se puede observar que la misma idea de Internet sobrepasa las posibles definiciones que desde una perspectiva mecanicista se podría dar, mediante las nociones rígidas de espacio y tiempo. ${ }^{3}$ Estas dos visiones de ver al mundo, mecanicistas y organicistas, nos proporcionan imágenes muy diferentes de cómo se puede ayudar a los alumnos a integrar las TIC en los centros, tal y como venimos argumentando. ${ }^{4}$

La visión Mecanicista se basa en los conceptos de tiempo, localización, y proceso pero éstos nos limitan y hace que utilicemos las TIC para realizar tareas educativas más tradicionales mientras que con una visión más organicista enfatizamos los procesos y como éstos se pueden integrar de maneras muy diferentes. De esta forma, nos enfrentamos a un dilema pedagógico, especialmente cuando nos referimos a las personas que no se sienten muy confortables utilizando las TIC. ${ }^{5}$ A continuación presentaremos diferentes maneras de trabajar con la tensión de la formación del profesorado entre una visión mecanicista y

${ }^{3}$ Es obvio que hay componentes mecanicistas al tratamiento de la noción de Internet, y nosotros no lo negamos. Es cierto que los ordenadores los localizamos físicamente en coordenadas de tiempo y espacio. Pero la naturaleza de Internet es tal que las explicaciones mecanicistas no llegan a alcanzar toda la profundidad del concepto.

${ }^{4}$ Una anécdota ilustra como esta visión mecanicista puede bloquear la experiencia del aprendizaje. Un profesor impartiendo un curso a distancia de manera colaborativa, dejó de participar en un momento dado porque había oído que el servidor, localizado fuera del centro donde trabajaba, no podía garantizar que la información fuera tan segura y fácilmente disponible que cuando lo tenían localizado en su propio centro. Su visión más mecánica enfatizando por encima de todo la noción de tiempo y espacio le impidió ver la parte más orgánica, Internet.

${ }^{5}$ No hay duda que se neces ita una visión mecanicista para poder funcionar a un nivel inicial. Zhao se refiere ha estas habilidades básicas cuando dice (2002: 491) que los profesores no necesitan " saber como funcionan las redes de ordenadores o saber como llegar a instalar un programa, pero es importante el entender las posibilidades de algunas tecnologías." El dilema que nos enfrentamos es que Internet presenta una dualidad: su función es esencialmente orgánica, pero las partes posibilitan que su función sea mecanicista. Y es la parte mecanicista la que es compatible con las imágenes más tradicionales de transmisión, repetición y memorización. Es decir, que en los primeros estadíos de aprendizaje es natural la tendencia a aprender de manea transmisiva, o si se prefiere más mecánica. Sin embargo, a los alumnos les cuesta ir más allá de este modelo mecanicista por sí solos. 
otra organicista. Hemos seleccionado un ejemplo muy concreto de la integració de las TIC, dentro de lo que es un curso optativo en la construcción de páginas web.

\section{LAS WEBS INTEGRADORAS}

Es importante recordar que nuestro interés en presentar el ejemplo específico de la construcción de páginas web no es tanto nuestro objetivo principal como el de ayudar a la formación del profesorado a pensar en otras maneras de integrar las TIC dentro del currículum. Así entonces, cuando uno revisa algunas de las páginas web de los centros educativos existentes en Internet, podemos ver como una explicación mecanicista puede orientar a mostrar, lo que vemos,

- Se muestra información estática que añade poca sustancia a lo que tendríamos acceso de igual manera sin una web,

- Ausencia de los objetivos educativos concretos,

- Ausencia de la presencia de los trabajos de los alumnos (a excepción de alguna propuestas específicas)

- Dificultad por encontrar "links" con otras páginas educativas relevantes,

- Contienen gráficos, que aunque aportan creatividad, no es tan obvia la aportación adicional dentro de la función de la web,

- Muchas veces, son profesionales, Web Masters los creadores, profesores de tecnología pero con relativa escasez de trabajo colaborativo entre profesorado y alumnos, $y$

- Normalmente las webs son monográficas de cada curso escolar perdiendo así el sentido integrador de todo el centro.

Estas características enfatizan el aspecto "técnico" de las TIC más que el buscar el sentido orgánico, por lo que hace al concentrarse en el proceso de las experiencias de los alumnos, pudiendo entonces alcanzar el sentido de integración intelectual y utilizar el término de Rodenburg (1999) "contexto apropiado". Típicamente, las webs que hemos observado no buscan la integración del contenido ni el proceso de su desarrollo y contribuyen mínimamente el sentido orgánico de conexión y coherencia interna. Más concretamente, no parecen disfrutar del potencial global que las TIC pueden aportar al aprendizaje de los alumnos. Como Rodenburg (1998) sugiere, la construcción de las webs de los centros es muy a menudo "fragmentada" o "jerárquica” añadiendo, que no sugiere un ejemplo de integración en el curriculum.

Con estas observaciones y la idea de integración en nuestras mentes, empezamos a pensar en cómo podríamos contribuir para mostrar experiencias que ayudaran a la formación del profesorado a pensar en términos de integración realistas. ${ }^{6}$ Nos planteamos la naturaleza orgánica de Internet lo cual nos ayudó a

${ }^{6}$ Hay tres perspectivas teóricas relevantes para nuestro trabajo: (1) La articulación de nuestra pedagogía proviene del trabajo Schön $(1983,1987)$. El concepto de Schön sobre "las conversaciones con la situaciones," "talkback," "move testing experiments," nos han ayudado en la articulaciones de nuestras reflexiones en el momento que trabajábamos con alumnos sin experiencia docente y basados en el modelo transmisivo durante los años de enseñanza y aprendizaje. (2) El enfoque general también has seguido los principios del Aprendizaje Basado en Problemas, ABP (e.g., CTGV, 1993). A lo largo de 6 años hemos ido utilizando simulaciones de situaciones en centros para que los alumnos pudiesen tener practicas reales para enriquecer su conocimiento. Los principios del ABP van perfectamente alienados con las prácticas reflexivas y los enfoques constructivistas de la enseñanza. (3) Nuestra idea de web para el aprendizaje (Álvarez and Kilbourn, 2003, 2004) siguió la idea Pepperiana del tratamiento de la visión del mundo organicista. Integración, conexión y coherencia interna como conceptos centrales en Organicismo han guiado nuestro pensamiento y las tareas para que los alumnos pudiesen trabajar de manera colaborativa - tareas que les permitieron plantearse conceptos educativos 
pensar en cómo, dentro del contexto educativo, podríamos buscar sistemas de conexión con experiencias relevantes y enriquecedoras para los alumnos. El hecho de trabajar en un programa de formación inicial de profesorado en una universidad Española, ayudó a concretar los esfuerzos. La idea era empezar con un grupo reducido de estudiantes lo cual nos podría permitir trabajar cómodamente, por este motivo se escogió un curso optativo. La idea central, que en un principio se veía más como un puzzle, era de qué manera podríamos trabajar con los alumnos para facilitarles la adquisición de habilidades básicas para el manejo de las TIC, al mismo tiempo y más importante, que aumentásemos el potencial para que un futuro, ya siendo profesores, pudiesen pensar en las TIC como una parte más que integra el curriculum en lugar de un elemento al que hay que añadir después. Pensamos abordar estos elementos con que lo llamamos las webs integradoras (coherent websites) ${ }^{7}$ lo cual pareció un buen punto de partida.

El camino a seguir se fundamentaba en las discusiones que íbamos manteniendo, aunque los pasos del proceso no se encontraban previamente planteados. Términos como ahora "conexión", "link", "integración”, se iban repitiendo de manera continúa a medida que avanzábamos. Particularmente, la idea de presentar las finalidades educativas en primer plano y dejar en un segundo plano las filigranas tecnológicas. El término, Webs integradoras, deriva directamente de una visión organicista, y era central para trabajar con este grupo de alumnos en la formación del profesorado. La idea fundamental de estas webs era el poder ayudar a los alumnos a participar desde el principio a construir un proceso en el cual tanto la información como la búsqueda de finalidades educativas estaban marcadas como principales actores. $^{8}$ En nuestras discusiones acordamos que las cualidades integradoras de estas webs debían plantear definiciones concernientes al proceso, contenido, y estructura:

- Proceso: El intento de facilitar que tanto los profesores como sus alumnos trabajaran conjuntamente en las webs, ayudó a nuestros alumnos a entender hasta dónde queríamos llegar con la idea de colaboración. Las conexiones orgánicas debían de producirse no solo con las informaciones sino también con as personas implicadas a la vez que se trabajaban elementos tanto educativos como más técnicos. Los elementos técnicos se esperaban que se iban a desarrollar lentamente durante todo el proceso, pero sabíamos que los aspectos educativos necesitaban más dedicación por nuestra parte, tales como "qué objetivos te plantearías para tu web?". En resumen, el proceso a seguir debía enfatizar el aspecto colaborativo de todas y cada una de sus partes.

- Contenido: El contenido de estas webs necesitaba que los alumnos se implicaran desde el principio de manera muy activa buscando finalidades educativas y sus implicaciones directas en el curriculum. Como cualquier web, dispondría de diferentes tipos de información, pero esta debía ir más allá de representar un listado de elementos, que es lo que algunas veces sucede con otras webs. El contenido necesitaba la participación de profesores y alumnos desarrollando tareas comunes y actividades donde el objetivo final fuese el compartir significados.

relevantes para la vida diaria de un profesor que iban mas allá del memorizar información y más allá de repetir una tarea en la web. Hemos argumentado que el modelo transmisivo de enseñanza y aprendizaje enfatiza el aspecto de "herramienta" y obvia la oportunidad de proporcionar experiencias interesantes para los alumnos que son más coherentes e intelectualmente más atractivas hacia la integración (Rodenburg, 1999).

7 Álvarez, I and Kilbourn, B. "Coherent Websites: Implications for Schools and Teacher Education," American Educational Research Association, Chicago, April 2003.

${ }^{8}$ Creemos en el potencial de las webs integradoras para ayudar a la posible creación de una "comunidad de aprendizaje" similar al Knowledge Forum (Scardamalia, 2002). 
- Estructura: Una web integradora es en realidad un conjunto de webs que están conectadas por su contenido y que se accede mediante una principal a la que llamamos la web de la escuela, (ver Fig.4). Como sucede con otras webs, las webs integradoras debía facilitar el movimiento interno de su conjunto.

Nuestra experiencia trabajando en la Formación Inicial del Profesorado sugiere que aunque los alumnos no se sientan muy confortables utilizando las TIC, pueden llegar a aprender de una manera que les abra la puerta para continuar explotando su potencial con posterioridad. A continuación presentamos la experiencia con el trabajo de nuestros alumnos, destacamos el trabajo de cuatro de ellos (Montse, Rosa, Patrícia, y Tomás-pseudónimos) los cuales empezaron con un planteamiento bastante tecnófobo. Explicitamos tanto el proceso como el resultado siendo éstos los elementos principales de igual manera como si se tratase de un estudio de caso que ofrece datos sobre cómo mejorar el resultado. El proceso que se describe a continuación muestra el trabajo de los alumnos a lo largo de 10 sesiones $^{9}$ :

\section{Sesión \#1}

Durante la primera sesión se les pasó un cuestuario con la idea de conocer sus experiencias anteriores con el uso de las TIC, particularmente con el manejo de Internet. Lo que obtuvimos fue que no tenían mucha experiencia, que muchas veces no entendían porque podían perder el documento con el cual habían trabajado con tanto esfuerzo, etc., lo cual hacía que no dedicasen mucho tiempo a explorar aplicaciones o a navegar por Internet. El uso del procesador de texto era la aplicación con la cual se sentían más cómodos y la que más a menudo utilizaban para los trabajos. Sin embargo, no compartían un mínimo vocabulario práctico sobre términos como archivo, escritorio, extensiones de archivos, etc. En este momento ninguno de ellos sabían o podían reconocer con el nombre de una extensión si se trataba de una imagen o de un texto. Después de conocer sus ideas, se pasó a presentar el curso, el cual se vio como la posibilidad de construir una web integradora donde cada uno de ellos jugaría el rol de un profesor responsable de un curso escolar.

\section{Sesión \#2}

En esta sesión empezamos a presentar el programa que nos ayudaría a construir las webs. Cuando esta presentación se hace con alumnos un tanto incómodos con las TIC es siempre difícil ver hasta qué punto se da la información necesaria o se da demasiada información en las primeras sesiones, lo cual contribuye en gran medida a mantener alto su nivel de ansiedad. La idea de continuar pero de la forma más sencilla y atractiva, al mismo tiempo sin provocar una lluvia de datos difíciles de digerir al principio. Aunque este curso se había diseñado con el objetivo de la construcción de webs, queríamos enfatizar la parte pedagógica por encima de la tecnológica en todo momento. De esta manera, las razones por escoger un programa determinado eran: que fuese sencillo, disponible y gratuito. Esto no solo nos ayudaría a avanzar rápidamente y romper el hielo de las primeras sesiones (que tienden a ser las más problemáticas), sino que el hecho de escoger un programa que estaba a su alcance en cualquier momento, al mismo tiempo para cualquier centro (debido a su gratuidad) hizo que mereciera la pena el intentarlo, no solo para los objetivos

\footnotetext{
${ }^{9}$ Los datos en los cuales nos basamos para la discusión provienen de cuatro fuentes diferentes: 1) pre/post notas de clase del profesor que impartía el curso (un de los autores de este artículo), 2) el cuestionario pasado a los alumnos el cual se centró en las ideas y conocimientos previos de los alumnos sobre sus experiencias, tanto positivas como negativas, durante la construcción de la web para el aprendizaje, 3) las webs integradoras creadas por los alumnos y, 4) reconstrucción de vignettes de los hechos más significativos del proceso de enseñanza y aprendizaje.
} 
del curso en si, sino para su futuro. El programa utilizado fue las plantillas de Yahoo en (http://geocities.com). Al mismo tiempo, se les pasó a los alumnos un material complementario para poder acceder a las plantillas de manera autodidacta.

Lo primero que hicimos fue darnos de "alta", esto significa crear una identidad ("user-name") y una contraseña ("password) de tal manera que podían acceder al programa y las plantillas. El hecho de poder empezar a trabajar sin tener mayor nociones, hizo que les ayudara a mantener la atención y al poder ver hasta dónde podrían llegar. En la primera prueba que se hizo, escribieron sus datos personales, pero al mismo tiempo se les pidió, siguiendo la modalidad de role-play, que empezaran a pensar en qué curso escolar les gustaría trabajar y qué tema del currículo les gustaría desarrollar. Así, se empezó a ver que teníamos dos grupos: Montse en Primaria con la especialización en ciencias, y Tomás en Inglés como lengua extranjera. Mientras, Patricia y Rosa en Infantil, las dos en matemáticas. Una vez que teníamos todos estos datos, se grabó lo que constituiría el primer intento de web a la que llamamos "introducción.html". Hay que recordar que esta era solo su segunda sesión y ya habían empezado a esbozar la primera página cada uno de ellos, con el rol de profesor que habían escogido. Al salir de clase, Rosa era todo sonrisas y dijo: "No me lo puedo creer-Ya tenemos una página web!"

\section{Sesiones \#3 y \#4}

Al empezar esta sesión retomamos las preguntas del cuestionario inicial por lo que se refería al vocabulario de conceptos básicos que empezaban a ser necesarios a medida que íbamos avanzando en la construcción de las webs, concretamente lo que se referían a las imágenes. Después de algunas demostraciones y prácticas empezamos a abordar otro tema importante que era crear un sentido de trabajo colaborativo entre ellos. Así, se les pidió, que era necesario el compartir los recursos y las dudas de manera que se empezaron a enviar por email todos estos elementos.

Como se puede observar el curso se iba desarrollando entre el aprender y compartir habilidades específicas con la colaboración en el planteamiento de tareas concernientes con el curriculum, es decir, entre actitudes mecanicistas con organicistas.

\section{Sesión \#5}

Hasta esta sesión los alumnos habían empezado a crear un intento de colaboración entre ellos. Aunque cada uno trabajaba desarrollando su curso y tema de manera independiente, al mismo tiempo se iban intercambiando elementos entre ellos, por ejemplo cada uno de ellos tenia disponible la primera web de sus compañeros. Es en esta sesión cuando pasamos a crear lo que representaría la segunda web donde se iba a desarrollar el tema que querían presentar en su curso. La selección de un tema relacionado con las líneas básicas del curriculum no fue una actividad sencilla, sino más bien un desafío en sí mismo, así empezaron a surgir muchas dudas relacionadas con el curriculum y la relación de enseñanza y aprendizaje. Esta tarea ya la habían experimentado en otros cursos de su formación. Así fue que Rosa escogió para trabajar "los cuadrados" (infantil), Patricia el concepto de "pequeño y grande" (infantil), Montse el "sistema respiratorio", y Tomas "pronombres posesivosen Inglés". Al terminar la sesión ya habían finalizado con el trazado general de la segunda web, la grabaron todos con el nombre de (tema.html). Uno de los elementos ha destacar fue que los alumnos pudieron ver la relación entre las dos webs así como la integración, desde el principio con el curriculum escolar. 


\section{Sesión \#6}

Al principio de esta sesión se les quiso recordar que uno de los objetivos a trabajar en este curso era el trabajo colaborativo para realizar las webs integradoras. Esto seguía la idea inicial del role-play de un centro y su rol como profesores en la construcción de sus cursos, así que se les pidió que empezaran a pensar en maneras con las cuales ellos podrían colaborar estrechamente. De esta manera empezaron con la idea de intercambiarse materiales que iban leyendo y fotografías que podían ayudar a los compañeros a ofrecer una imagen mucho mas creativa.

Al mismo tiempo, es importante volver a insistir en la ausencia de experiencia docente. El hecho de utilizar el role-play empezó a dar sus primeros frutos al mismo tiempo que se les pidió que escribieran en tres paginas una reflexión sobre la relación entre los siguientes elementos: tecnología, enseñanza y aprendizaje, profesor. Para poder empezar con la tarea se les presentó un ejemplo en términos muy similares Álvarez (2000: 21-22):

Mi interés por ver como funcionaban las cosas empezó a tener forma cuando mi hermano y yo descubrimos los ordenadores. Desde el principio me fascinó su funcionamiento. Me entusiasmaba el poder desmontar y volver a montar una pieza e intentar arreglar algún que otro fallo en el sistema. Pero, al mismo tiempo que yo disfrutaba, también me podía dar cuenta de porqué algunos de mis amigos no compartían el mismo entusiasmo. De alguna manera, la fascinación por los ordenadores (y como funcionaban) era algo privado, pero cuando empecé a cursar clases de tecnología en secundaria empecé a conectar con las experiencias de mis amigos...

Ver a mis compañeros paralizados delante del ordenador fue algo que me impresionó mucho. Era un temor que nunca había conocido. El hecho de que el ordenador se "colgara" era mucho más que una metáfora para ellos y me llevó tiempo poder llegar a entender lo que les pasaba. La parte mas importante para mí fue, otra vez, cuando me encontraba ayudando a los compañeros, igual que había hecho anteriormente con algunos de mis juguetes siendo pequeña. En las tardes, después de las clases, subíamos al aula de ordenadores y les mostraba algunas maneras para poder arreglar algunos problemas que iban surgiendo. En retrospectiva, están experiencias fueron claves en mi desarrollo personal.

Los trabajos sobre la reflexión promovió un debate muy interesante entre ellos. Por ejemplo, Montse escribió que ya desde muy pequeña le habían interesado los ciclomotores y cuando tuvo la oportunidad de ahorrar dinero, se compró uno. Montse, en un principio no se sentía cómoda compartiendo experiencias sobre ciclomotores, ni si quiera la idea de intentar desmontar el ciclomotor para limpiarlo, como hacían sus amigos, aunque le encantaba su ciclomotor, no le interesaban las partes mecánicas ni como funcionaban, pero poco a poco, después de oír muchas cosas de sus amigos, empezó en su casa a desmontar algunos tornillos del chasis para poderlo limpiar mejor por dentro. Poco a poco empezó a sentirse mas cómoda con su ciclomotor y esperaba que algo parecido ocurriera con su ansiedad inicial con los ordenadores. Como futura profesora, esperaba ayudar a sus compañeras a aprender conjuntamente.

\section{Sesión \#7}

En esta sesión se les pidió que leyeran con atención el artículo de Norton (2000) sobre la experiencia de 4 profesores integrando las TIC en un centro de Australia. Al igual que hasta ahora, seguimos la técnica de role-play adoptando cada uno de los roles. Así podríamos comentar como cada uno de ellos reaccionó delante de la misma oportunidad. El objetivo era proporcionarles un ejemplo para 
suplantar la ausencia de experiencia docente en el programa de formación inicial, una versión de lo descrito entre la tensión mecanicista-organicista de la primera parte de este articulo-De qué modo podemos aportar experiencias reales a nuestros alumnos? En la estructura de este programa, en particular, era difícil para los alumnos disponer de experiencias reales en los centros educativos mientras cursaban esta asignatura. Así, de esta manera el artículo (Norton, 2000) venía a suplir esta ausencia. La idea general del artículo era la percepción en la noción de enseñanza y aprendizaje que tenemos y que opera en cómo utilizamos las TIC, siendo una opción la de incorporar las tecnologías sin un contexto significativo o como vehiculo integrador para ampliar las posibilidades educativas de nuestros alumnos. De alguna forma, el artículo venía a reflejar las discusiones entre enfoques mecanicistas y organicistas sobre el uso de las TIC. En el artículo, dos profesores (Peter y Eva) se situaban en un enfoque "tradicionalista" y, en aquellas ocasiones donde utilizaban las TIC, era solo para ilustrar un enfoque tradicional de aprendizaje de las matemáticas, de manera memorística. Es decir, era difícil ver lo que se conseguía con sin las TIC. El tercer profesor (Mary) se desmarcó partiendo de un enfoque más orgánico al utilizar las TIC para explorar elementos conceptuales de las matemáticas que podían ser complejos sin ellas. Mary expresó su preocupación varias veces por la ausencia de colaboración con sus colegas en el centro por mantener posturas divergentes de lo que significa enseñar y aprender y porque su interés de explorar nuestras estrategias con las TIC. La discusión que promueve este artículo contrasta los diferentes enfoques de enseñanza y las TIC:

Los profesores describieron lo que representa mostrar una imagen de transmisión/absorción con lo que significa enseñar y aprender (lo cual se articuló en la pedagogía centrada en el profesor) reconocieron el gran potencial que tenían los ordenadores para calcular ... Sin embargo, Peter y Eva también apuntaron que el uso de los ordenadores en el aula aportaba efectos negativos. Por ejemplo, no facilitara que el alumno practicase las habilidades básicas así como los procedimientos que son la esencia del aprendizaje de las matemáticas ... (p.104)

La imagen que Mary tenía sobre el aprendizaje se acercaba más a la idea constructivista de lo que significa enseñar y aprender. Su pedagogía reflejaba esto al utilizar elementos según una pedagogía centrada en el alumnos. Mary vio a los ordenadores como la herramienta mediante la cual los alumnos podían construir significado matemático y explorar la naturaleza matemática de los elementos. Esto es así, ella creía que los alumnos podían utilizar los ordenadores para ayudar a plantear los conceptos matemáticos así como para calcular y practicar demostraciones. (p.105)

Los alumnos leyeron el artículo con mucho interés y se les pidió que intentasen ver las tensiones entre orientaciones mecánicas y organicistas expresadas por los cuatro profesores. No fue muy difícil el poder ver las limitaciones que una visión transmisiva aportaba en la integración del ordenador en el aula.

\section{Sesiones \#8 - \#10}

En las tres últimas sesiones los alumnos finalizaron creando las webs integradoras. Los pasos seguidos se encuentran ilustrados con la ayuda de los diagramas abstractos que representan cada una las webs y el proceso seguido. Es importante volver a recordar que al principio del curso, estos alumnos partían de una base incómoda al pensar en el uso de las TIC y que incluso dos de ellos se podían ubicar en una visión más tecnófoba. Sin embargo, a lo largo de todo este proceso empezaron rápidamente a sentirse cómodos con su uso, en parte creemos al hecho de los esfuerzos continuos por integrar el curriculum en todas las sesiones en lugar de centrarnos solo en la parte más técnica. Al principio de la sesión octava los 
alumnos ya habían completado con éxito dos webs de manera individual, aunque compartiendo colaborativamente recursos e ideas. La primera web "introducción.html", era la más sencilla, y respondía al deseo de presentación de cada uno de los alumnos en el rol de profesor, indicando sus intereses pedagógicos; en la segunda web, "temas.html", se trataba de explicar el porqué de la elección de cada tema y el porqué se basaban en un curso específico (cuadrados, tamaño, sistema respiratorio, pronombres posesivos). Entonces aprendieron la tarea de unir las dos webs, tal y como aparece ilustrado en la Figura 1, y así practicaron como nos podíamos mover entre ellas de manera ágil.
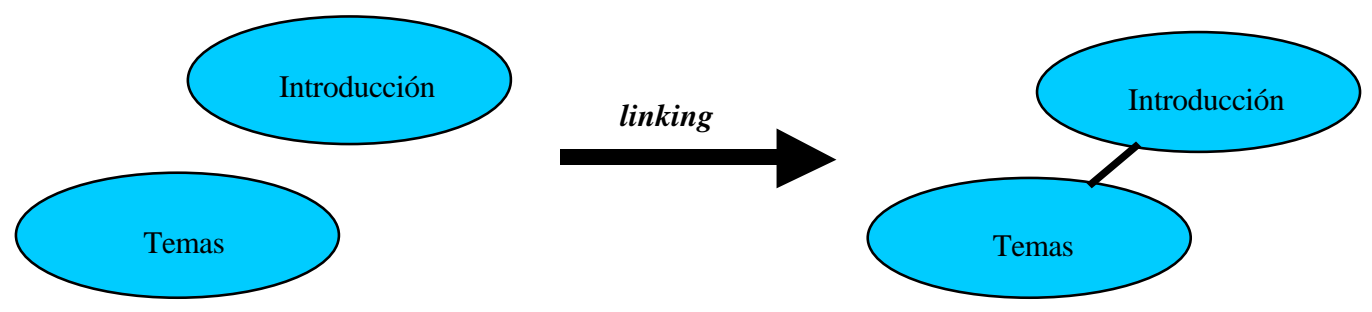

Figura 1

Después de esa fase más mecánica pasamos a otra un tanto más crítica. Cada alumno había desarrollado una tercera web (actividades.html), que incluía las actividades, en relación al tema escogido que los alumnos deberían realizar para poder aprender el tema en cuestión. Dos cosas sucedieron en este momento y que son importantes destacar. La primera, después de la creación de la tercera web, nos planteamos como grupo de profesores el revisar las webs realizadas de manera crítica. Se les pidió que revisasen sus webs y las de sus compañeros teniendo en cuenta la discusión que provocó la lectura de Norton (2000). En esta discusión se dieron cuenta de que cada uno de ellos había realizado una web tradicional. Las actividades que se habían planteado no explotaban el carácter orgánico del medio. Así, lo que habían hecho se podía haber realizado sin el uso de la tecnología.

Es en este momento cuando como grupo empezaron a trabajar todos entre ellos de manera colaborativa para ver de manera conjunta como se podía solucionar. Por ejemplo, empezaron con el tema de los "cuadrados" y empezaron a preguntarse de qué forma podían facilitar que en una actividad se implicaran los alumnos a los cuáles iba dirigida en lugar de ser los destinatarios pasivos. Una posibilidad era que los alumnos buscasen en Internet imágenes que tuvieran como denominador común el hecho de una forma cuadrada. Mientras que esta actividad se podría realizar perfectamente sin el uso de ordenador, por ejemplo, utilizando revistas, periódicos, papel y lápiz, era importante lo que estaba sucediendo, ya que estaban empezando a pensar mas allá de las imágenes tradicionales entre tecnología y aprendizaje al mismo tiempo que empezaban a explorar las posibilidades más orgánicas. Fue en este momento cuando también empezaron a comprender que ayudándose entre ellos era mucho más efectivo al mismo tiempo que suponía un esfuerzo en el que todos se beneficiaban por igual.

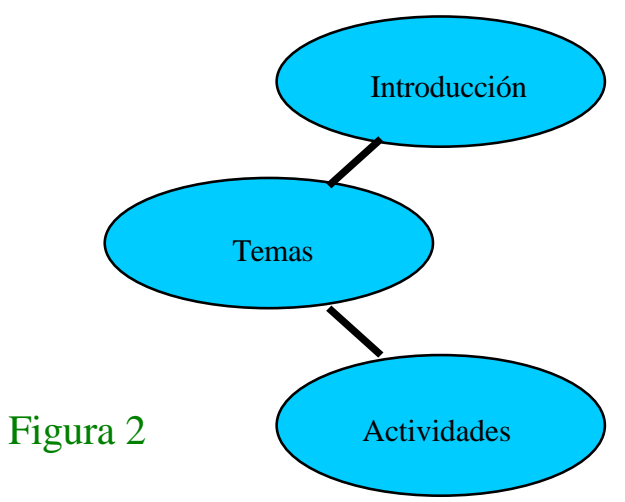


La segunda, es que los alumnos empezaron a utilizar las habilidades que habían aprendido en el curso y empezaron a relacionar y unir la web de las actividades con la del tema. Una vez realizado este paso, cada alumno tenía una estructura simbolizada en la Figura 2.

En este momento teníamos un conjunto de cuatro grupos de webs interconectadas (Figura 3). Aunque, la web de las actividades se había realizado de manera colaborativa, cada uno de ellos la mantuvo asociada y representada independientemente:
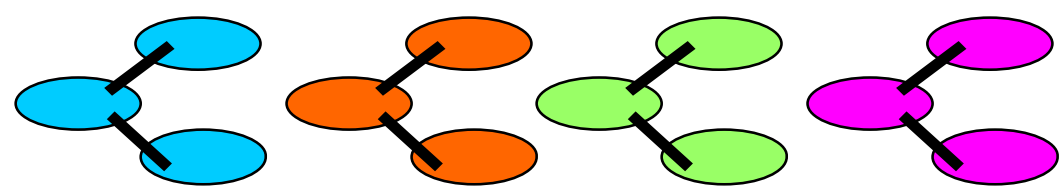

Figura 3

Manteniendo la idea inicial de que los alumnos (profesores) trabajan en el mismo centro y cuyo objetivo era la creación de una comunidad de aprendizaje, empezaron a discutir el tipo de "links" que permitiría a otros profesores acceder a sus webs. Decidieron que necesitarían tener una página central a la que llamaron escuela (escuela.html) a la cual todas las webs creadas irían a converger. No es extraño que la producción de la última web llevó considerable tiempo y discusión, pero lo más importante es que estos alumnos trabajaron colaborativamente y fueron más allá del mero hecho de aportar una lista de nombres. Además, cada conjunto de webs estaban todas unidas (representadas en la Figura 4), lo cual aportaba mayor coherencia interna al conjunto elaborado colaborativamente..

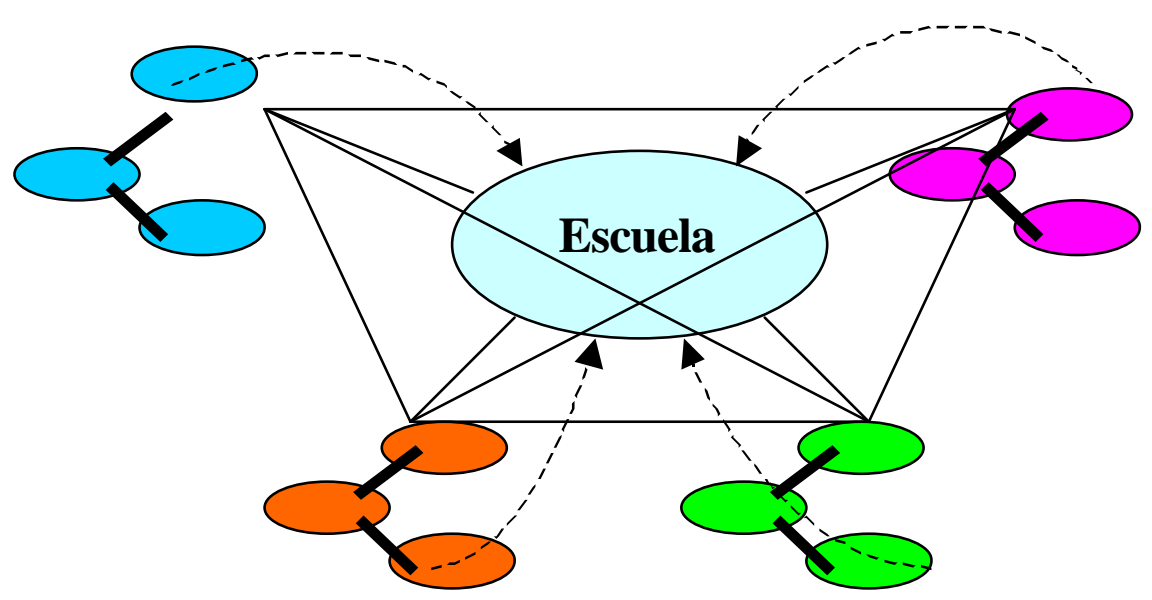

\section{CONCLUSIONES}

Figura 4

Partimos de la base que una de las razones por las cuales es difícil integrar las TIC en el curriculum es el hecho de que en la formación del profesorado es difícil ir más allá de repeticiones y provocar el interés por la integración en los centros. De igual manera, los alumnos no disponen de experiencias con las cuales puedan practicar de manera realista. Si se pretende conseguir la integración, entonces nuestro objetivo es el de provocar el debate dentro de la misma formación del profesorado. Hemos planteado que la construcción de las webs integradoras es un ejemplo para mostrar a los alumnos, en su formación inicial, lo que podría ser su integración en el aula, utilizando el currículo escolar. Nuestra experiencia con un grupo 
limitado de alumnos sugiriere que pueden aprender de manera significativa y que ello puede abrir una puerta para el potencial de las TIC en los centros.

Después de que el curso concluyera llegamos a las siguientes conclusiones tentativas. Es importante que lo que conseguimos con estos alumnos se pueda llegar a explorar con más casos y de manera más extensa. Sin embargo, es muy interesante el articular las conclusiones a las que hemos llegado. Estamos satisfechos con los resultados obtenidos en este proceso. Basado en las evidencias aportadas (incluyendo la naturaleza y la calidad de las webs) sentimos que estos alumnos disfrutaron de una experiencia colaborativa que les permitió pensar sobre la construcción de webs de manera que hubiera sido más difícil si se hubieran hecho de manera individual. Además, desprendido de las discusiones, era evidente que comprobaron la diferencia entre los esfuerzos colaborativos y los individuales y que ello tenía una repercusión muy importante para dar los primeros pasos en la construcción de comunidades de aprendizaje, evitando el trabajo aislado de los profesores. Dado el contexto, es incierto si lo que aprendieron traspasara los limites de un curso en su formación, pero sabemos que algunos de ellos empezaron a utilizar webs para otros trabajos dentro de su formación y que comentaron la disponibilidad de arriesgarse posteriormente, igual que lo hicieron como parte de este curso. ${ }^{10}$

Como resultados del trabajo colaborativo (las propias webs integradoras), es necesario recordar que empezaron con una base mínima de conocimientos. Estamos satisfechos con el resultado final y que dadas las tres características para la construcción de una web integradora (proceso, contenido, y estructura), diríamos que sí que llegaron a construir webs que tuvieron en cuenta estas características. Que los alumnos empezaron el curso con una gran motivación por aprender al mismo tiempo que intentar tratar la ansiedad que les producía trabajar con el ordenador. Al final del curso construyeron una web de una escuela, aunque modesta. Todas las webs se iniciaron partiendo de los objetivos pedagógicos para después pasar a los aspectos técnicos. El nivel de coherencia era limitado por el hecho de que todos ellos partieron del trabajo de temas diferentes, preocupación que queremos trataran en posteriores intentos. De lo que estamos más satisfechos, no es tanto de la mera construcción de la web de centro (aunque ello fue unos de los objetivos), sino de las conversaciones que tuvieron la oportunidad de experimentar planteándose problemáticas educativas realistas.

Desde nuestro punto de vista, la historia a explicar en este proyecto se basa en lo que aprendimos con estos alumnos en su formación y los pasos específicos que hemos ido promoviendo de manera colaborativa, con un aprendizaje constructivista sobre la integración de las TIC en el currículo. Nuestro objetivo es el de aumentar las posibilidades de los alumnos para poder integrar las TIC de manera colaborativa y con actitudes constructivistas cuando lleguen a tener sus propios cursos, aunque sea una tarea lo suficientemente desafiante al principio. Varios elementos emergieron de los datos cualitativos:

- Consideramos nuestro éxito relativo por lo que hace referencia al aprendizaje de habilidades técnicas en los alumnos al mismo tiempo que nos centramos y preocupamos por los temas educativos en la construcción de las webs (permitiendo que la tecnología no nos impidiera andar);

- Los alumnos empezaron a trabajar de manera colaborativa y productiva;

\footnotetext{
${ }^{10}$ Básicamente esto nos apuntó hacia estudios longitudinales.
} 
- Partir con alumnos que se sitúan en una línea tecnófoba ha sido gratificante por el resultado final obtenido;

- La ausencia de experiencia docente de estos alumnos continua siendo un problema serio (acentuado en gran parte por el hecho de que muchos de estos cursos de iniciación a las TIC están situados al principio de su formación, lo cual hace que no dispongamos de oportunidades para garantizar su experiencia docente. Sin embargo, estamos satisfechos por el enfoque basado en problemas, con lo cual nos ayudó a presentar y ver diferencias en enfoques, así como los alumnos disfrutaron de la oportunidad de discutir elementos claves que los docentes experimentados se enfrentan diariamente;

- A pesar de estos resultados positivos, la presión por una "cultura de transmisión" no deja de ser un desafío constante y laborioso

- Finalmente, la ausencia de experiencia docente junto con la cultaura transmisiva hace difícil que los alumnos se puedan dedicar a explorar nuevas ideas y/o planteamientos.

Ayudar a estos alumnos a vivenciar un ejemplo de cómo integrar as TIC al construir las webs integradoras es un primer paso de lo que representa un problema de dimensiones superiores, pero ayuda a ver otras aproximaciones igualmente válidas y ampliamente satisfactorias. Las actividades que realizaron los alumnos requirieron que trabajasen de manera conjunta y colaborativa.

\section{REFERENCIAS}

ÁlvarEZ, I., and KILBOURN, B., (2004) Website Construction as Learning Tools, Teoría de la Educación, Educación y Cultura en la Sociedad de la Información, July-August, Vol 1, ISSN-1138-9737

ÁlvareZ, I. and KILBOURN, B. (2003) Coherent Websites: Implications for Schools and Teacher Education, American Educational Research Association, Chicago, April.

ÁlvaREZ, I., and KILBOURN, B., (2002). Mapping the information society literature: Topics, perspectives, and root metaphors. First Monday, $7(1) \quad$ January, At (http://firstmonday.org/issues/issue7 1/alvarez/index.html)

BECKER, H. (1994). How exemplary computer-using teachers differ from other teachers: Implications for realizing the potential of computers in schools. Journal of Research of Computing in Education, 26 (3), 291-321.

BEICHNER, R.J. (1993). Technology competencies for new teachers: Issues and suggestions. Journal of Computing in Teacher Education; 9 (3), 17-20.

Bereiter, C. \& ScARdamaliA, M. (2000). Process and product in Problem-Based Learning (PBL) research. In D.H. Evensen and C.E. Hmelo (Eds.), Problem-Based Learning: A Research Perspective on Learning Interactions (Mahwah, NJ: Lawrence Erlbaum Associates), 185-195.

BRAND, G. (1997). What research says: Training teachers for using technology. Journal of Staff Development, 19 (1), 10-13.

BRUNNER, C. (1992). Integrating technology into the curriculum: Teaching the teacher. Technical Report. N. 25, New York: Center for Teaching in Education. 
BRUSH T. (1998). Teaching preservice teachers to use technology in the classrooom. Journal of Technology and Teacher Education, 6 (4), 243-258.

Brush, T. igoe, A., Brinkerhoff, J., GlaZeWSK, K., Ku, Hy., \& SMITH, C. (2001). Lessons from the field: Integrating technology into preservice teacher education. Journal of Computing in Teacher Education, 17(4), 16-20.

CARR, L.L. (1992). Integrating technology into preservice education: Determining the necessary resources. Journal of Computing in Teacher Education, 9 (1), 20-24.

Cognition \& TEChNology Group At VANDERBilt (1993). Anchored instruction and situated cognition revisited. Educational Technology, 33(3), 52-70.

DUHANEY, D.C. (2001). Teacher education: Preparing teachers to integrate technology. International Journal of Instructional Media, 28 (1), 23-30.

ERTMER, P.A. (1999). Addressing first and second-order barriers to change: Strategies for technology integration. Educational Technology Research and development, 47 (4), 47-61.

FABRY, D.L. \& HIGGS, J.R. (1997). Barriers to the effective use of technology in education: Current status. Journal of Educational Computing Research, 17 (4), 385-395.

Falba, C.J., Strudler, N., Bean, T.W., Dixon, J.K., Markos, P.A., MCKInNeY, M., \& Zehm, S.J. (1999). Choreographing change one step at a time: Reflections on integrating technology into teacher education courses. Action in Teacher Education, 21 (1), 61-76.

Franklin, T., TURNeR, S., KarIUKL M., \& DURAN M., (2001). Mentoring overcomes barriers to technology integration. Journal of Computing in Teacher Education. 18 (1), 26-31.

HAUGLAND, S.W. (1999). What role should technology play in young children's learning? Young Children, 54 (6), 26-31.

KILBOURN, B., (1998). For the Love of Teaching. London, ON: Althouse Press.

MARTíNEZ, J. AND WOODS, M. (1995). The value and planned use of educational technology in higher education: Results of a faculty service needs assessment. College \& University Media Review, 2 (1), 25-28.

MUNDAY, R., WINDHAM, R., \& STAMPER, J. (1991). Technology for learning: Are teachers being prepared? Educational Technology, 31 (3), 29-32.

NORTHOVER, M. (1999). Developing a successful information technology competency strategy for faculty and staff. The Technology Source, January, 56.

NORTON, S., MCROBBIE, C., \& COOPER, T. (2000). Exploring Secondary mathematics teachers' reasons for not using computers in their teaching: Five case studies. Journal of Research on Computing in Education, 33 (1), 87-109.

PEPPER, S. C. (1942) World Hypotheses: A Study in Evidence. University of California Press.

Pierson, M.E. \& MCNEIL, S. (2000). Preservice technology integration through collaborative action communities. Contemporary Issues in Technology and Teacher Education, 1 (1), 189-199. 
RoDENBURG, D. (1998) Shifting perspectives in educational technology. The Technology Source. December.

RODENBURG, D. (1999a) Web-based learning: extending the paradigm. The technology source. Nov/Dec.

RODENBURG, D. (1999b) "Web-based learning: Extending the paradigm," The Technology Source. Nov/Dec, (http://ts.mivu.org).

RODRIGUEZ, S. (1996). Preparing preservice teachers to use technology: Issues and strategies. TechTrends, 41 (4), 18-22.

SALMON, G. It's not just the tool, but the educational rationale that counts. Ed-Media Meeting. Montreal, June 28, 2000 at (http://construct.haifa.ac.il/ gsalomon/edMedia2000.html)

ScARDAmaliA, M. (2002). Creative work with ideas: A luxury? Symposium: You say you want a Revolution...? Can New Technologies Enable Radically New Kinds of Learning? American Educational Research Association, New Orleans.

SCHÖN, D. (1983) The Reflective Practitioner. Basic Books, Inc.

SCHÖN, D. (1987) Educating the Reflective Practitioner. Jossey-Bass Inc., Publishers.

SCHRUM, L. (1999) Technology professional development for teachers. Educational Technology Research and Development, 47 (4), 83-90.

SIEGEL, J. (1995). The state of teacher training: The results of the first national survey of technology staff development in schools. Electronic Learning, 14(8), 43-53.

SMITH, R.A., HOUSTON, W.R., \& ROBIN, B.R. (1994). Preparing preservice teachers to use technology in the classroom. The Computing Teacher, 22 (4), 57-59

StRUDLER, N., \& WETZEL, K. (1999). Lessons from exemplary colleges of education: Factors affecting technology integration in preservice programs. Educational Technology Research and Development, $47(4), 63-81$.

SUGAR, W., (2002). Applying human-centered design to technology integration: Three alternative technology perspectives. Journal of Computing in Teacher Education, 19 (1), 12-17.

THOMPSON, A., SCHMIDT, D., \& HADJIYIANNI, E. (1995). A three year program to infuse technology throughout a teacher education program. Journal of Technology and Teacher Education, 3 (1), 13-24.

TOPP, N.W., MORTENSEN, R., \& GRANDGENETt, N. (1995) Building a technology-using facility to facilitate technology-using teachers. Journal of Computing in Teacher Education, 11(3), 11-14.

U.S.CONGRESS, Office of Technology Assessment. (1995) Teachers and technology: Making the connection (OTA-HER-616). Washington, DC. US Government Printing Office.

WETZEL, K. (1993). Teacher educator's uses of computing in teaching. Journal of Technology and Teacher Education, 1 (4), 335-352.

WILLIS, E.M. (1997). Technology: Integrated into, not added onto, the curriculum experiences in preservice teacher education. Computers in the Schools, 13 (1-2), 141-53.

ZHAO, Y., PUGH, K., SHELDON, S., \& BYERS, J., (2002). Conditions for classroom technology innovations. Teachers College Record, 104 (3), 482-515. 


\title{
Contactar
}

Revista lberoamericana de Educación

\author{
Principal OEI
}

